\title{
Dehydroepiandrosterone(DHEA) combining Metformin - Adjuvant Agents for Ovulation Induction in Poor Ovarian Responders
}

\author{
Chih-Chia Chang ${ }^{1}$, Yu Chang ${ }^{2}$, and YU Chang ${ }^{3}$ \\ ${ }^{1}$ Affiliation not available \\ ${ }^{2}$ Chang Hua Christian Hospital \\ ${ }^{3}$ National Taiwan University Hospital
}

July 1, 2020

\begin{abstract}
Objective: To investigate the impact of Dehydroepiandrosterone(DHEA) combining Metformin on IVF-ET outcomes in poor ovarian responders(PORs). Materials and Methods: This is a retrospective cohort study. There were 237 women undergoing IVF-ET cycles. A total of 106 cycles met the Bologna criteria (44.8\%) as poor responders in this study period. They were divided into two groups according to with or without supplementation. Results: The number of oocytes retrieved (3.5 \pm 2.5 vs. $1.5 \pm 0.8, \mathrm{p}=0.027)$, the clinical pregnancy rate(21.7 vs. $11.5, \mathrm{p}=0.008)$ and the live birth rate $(16.7$ vs. $8.7, \mathrm{p}=0.007)$ were significantly higher in the group with supplementation than in the group without. Conclusion: The adjuvant agents: DHEA and Metformin for ovulation induction seemed to improve the number of oocytes retrieved, clinical pregnancy rate and live birth rates in poor ovarian responders.
\end{abstract}

\section{Introduction}

The birth of the first IVF baby resulted from the transfer of single embryo from a spontaneous ovulation cycle by Steptoe and Edwards in 1978. Soon after this ground breaking event, controlled ovarian hyperstimulation $(\mathrm{COH})$ was introduced. The availability of a high number of oocytes facilitated the pregnancy rate of IVF treatment. However, after more than forty years of experience, there are still women who respond poorly to stimulation, resulting in only few oocytes at retrieval, a reduced number of embryos available for transfer and a poor pregnancy rate. The prevalence of poor ovarian responders among infertile women is reported to vary between $5.6 \%$ and $35.1 \%$ depending on differences in the definition of poor ovarian response but according to recent reviews, it seems to have slightly increased $(6,23)$. Though there might be numerous causes for the poor ovarian response. The European Society for Human Reproduction and Embryology (ESHRE) working group on poor ovarian response (POR) has finally given a common definition of "poor responder," where at least two of the following three features must be present: (a) advanced maternal age or any other risk factor for POR; (b) a previous POR; and (c) an abnormal ovarian reserve test.

In the field of assisted reproductive technologies great steps forward have been made in recent years in terms of clinical knowledge and technological development especially in IVF laboratories. One of the fundamental steps to success is still related to the number of oocytes retrieved after hormonal stimulation. In fact, with lower number of retrieved oocytes, fewer embryos are there to be selected and transferred and thus lower pregnancy rates per transfer and lower cumulative pregnancy rates per started cycle. Ovarian follicles mature over a period of approximately 2-4 months. Ovarian stimulation in IVF cycles has traditionally been focused on the stimulation of antral follicles, which develop during the last 2 weeks of this maturation process, to increase the number of mature follicles for oocyte retrieval. However, successful ovarian stimulation with gonadotrophins is limited by the requirement of the presence of multiple antral follicles (1). The stimulation 
and synchronization of earlier follicles prior to traditional ovarian stimulation may thus further improve IVF outcomes, particularly for poor responders (21). The follicles require about $6-8$ weeks after the initiation of androgen supplementation to achieve synchronization and become mature enough to respond to ovarian stimulation with gonadotrophins $(14,15,16)$. Based on this, many patients could potentially benefit from androgen supplementation(DHEA) beginning weeks or months prior to starting their IVF cycle.

The beneficial effect of metformin on ovulation induction with clomiphene in clomiphene-resistant and obese women with polycystic ovary syndrome was originally reported in 1998 (22). Metformin is a drug that affects metabolism and induces ovulation by reducing the circulating concentration of insulin. Onset of metformin is slow and gradual. To clinically improve ovulation, it may require up to 6 months of treatment with metformin.

In present study, we aim to investigate the impact of adjuvant agents: DHEA and Metformin on the number of oocytes retrieved, pregnancy rates and live birth rates in the poor ovarian responders. The differences in the effect of adjuvant agents on IVF outcomes between the aged and the younger poor responders were compared. This study could be helpful in determining the influence of DHEA and metformin on controlled ovarian hyperstimulation.

\section{Materials and Methods}

\section{Study population}

This is a retrospective cohort study. Patients were treated with routine practice of IVF from July 2016 to December 2018 at Mei-Tsun Reproductive Medical Center. The data was collected from medical records and the computerized database. The definition of a poor responder fits into at least two of the following criteria: (1) A previous episode of poor ovarian response ([?]3 oocytes) under a standard dose of medication; (2) An abnormal ovarian reserve with AFC (Antral Follicle Counts) $<5-7$ follicles or AMH (Anti-Mullerian Hormone) $<0.5-1.1 \mathrm{ng} / \mathrm{mL}$; (3) Women above 40 years of age or those with other risk factors for poor response such as previous ovarian surgery, genetic defects, chemotherapy, radiotherapy, and autoimmune disorders.

Patient characteristics including age, body mass index (BMI), basal AFC, and basal hormone levels were recorded. Clinical parameters including gonadotropin dosage, E2 levels at human chorionic gonadotropin (hCG) day, number of oocytes retrieved, fertilization rate, clinical pregnancy rate, and live birth rates were analyzed.

\section{Supplementation}

Each patient received dehydroepiandrosterone (DHEA 30mg, Wild Yam Root extract, VIVA Pharmaceutical Inc., Canada) $90 \mathrm{mg}$ daily from 3 months prior to starting IVF cycle until the day of human chorionic gonadotropin (hCG) trigger. Metformin (Metformin Hydrochloride $500 \mathrm{mg}$, Swiss Pharmaceutical Co.,Ltd , Taiwan ) was given to the patients $1500 \mathrm{mg}$ daily from 3 months prior to starting IVF cycle until a positive pregnancy test or menstrual bleeding appeared.

\section{IVF process}

Controlled ovarian hyperstimulation was performed using the short GnRH agonist protocol. Buserelin nasal spray (Buserelin acetate 10.5mg, Sanofi-Aventis Deutschland GmbH , Germany), 300ug three times daily, was administered from day 2 of treatment cycle until the day human chorionic gonadotropin (hCG) trigger. Ovarian stimulation was achieved the next day with $300 \mathrm{IU}$ dose recombinant FSH (rFSH: Gonal-f@) , Merck-Serono, Geneva, Switzerland, or Puregone@ B, Organon, Espanola S.A., Barcelona, Spain) and 150 IU dose hMG (Pergovaris , $150 \mathrm{rFSH}+75 \mathrm{rLH}$, Merck Serono, Aubonne, Switzerland, or Merional , 75 FSH+75 LH, IBSA Institut Biochimique S.A.,Switzerland ). The selection of the type of gonadotropin was individualized according to each patient's characteristics. Serial folliculometry and assessment of serum E2, P4 and LH levels were monitored every 1-2 days, starting from 3-5 days after stimulation and continued until two or more follicles reached $17 \mathrm{~mm}$ in diameter or when per-follicle E2 level reached $200 \mathrm{pg} / \mathrm{ml}$. Dual 


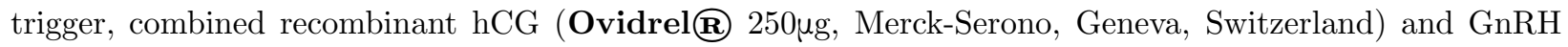
agonist (Lupro $2 \mathrm{mg}$, Nang Kuang Pharmaceutical Co, Ltd., Tainan, Taiwan), were administered. Oocytes were retrieved 34-36 hours after oocyte trigger under the guidance of transvaginal ultrasound. Intracytoplasmic sperm injection (ICSI) was performed in all patients to diminish potential fertilization failure. The best morphologically graded embryos from the stimulated cycle was selected for embryo transfer at 3 days after oocyte retrieval. Luteal support commenced from the second day after oocyte retrieval with Crinone 8\% Progesterone Vaginal Gel (Progesterone 90mg, Merck Serono, Hertfordshire, UK) andUtrogestan (Micronised Progesterone 100mg, Arich Enterprise Co., Ltd., Taiwan). A pregnancy test was carried out 15 days after embryo transfer. Once a positive pregnancy test was observed, progesterone was continued until 10-12 weeks of gestation. A clinical pregnancy was confirmed with the presence of a fetal heart beat at 7 weeks of gestation by ultrasonography. A live birth was defined as delivery after 24 weeks of gestation.

\section{Statistical analysis}

All data were analyzed using the commercially available software package SPSS (SPSS Inc., Chicago, IL, USA) and presented as mean \pm standard deviation or number (\%). Statistical analysis was carried out using Mann-Whitney $\mathrm{U}$ test for continuous data and $\chi^{2}$ test for categorical data. Differences were considered to be significant when $p$ value $<0.05$.

\section{Results}

A total of 106 cycles met the Bologna criteria (44.8\%) in this study period. Among these cycles, they were divided into group A with supplementation of DHEA combining Metformin $(\mathrm{n}=64)$ and group B without supplementation $(\mathrm{n}=42)$. There were no differences between the two groups regarding age, body mass index, basal follicle stimulation hormone concentration (FSH), basal estradiol concentration (E2), basal antral follicle counts (AFC), Anti-Müllerian hormone concentration (AMH) and Bologna criteria category.

The treatment cycle outcomes between the two groups were compared (Table 1). There were no significant differences in terms of stimulation duration, gonadotropin dosage, estradiol level on hCG day, and fertilization rate. Although not statistically significant, there is a trend of higher number of metaphase II oocytes retrieved $(2.1 \pm 1.5$ vs. $1.0 \pm 0.8, p=0.115)$ in group A than group B. Furthermore, the number of oocytes retrieved $(3.5 \pm 2.5$ vs. $1.5 \pm 0.8, p=0.027)$, the clinical pregnancy rate $(21.7$ vs. $11.5, p=0.008)$ and the live birth rates (16.7 vs. 8.7, $p=0.007)$ were significantly higher in group A than in group B.

\section{Discussion}

This is the first prospective study to evaluate the effectiveness of adjuvants with DHEA combining Metformin in poor ovarian responders (PORs) undergoing IVF. This study demonstrated that after supplementation pretreatment, the number of oocytes retrieved, the clinical pregnancy rate and the live birth rates significantly improved in poor ovarian responders. However, larger prospective studies and randomized controlled trials are necessary to confirm the possible benefit of this approach.

Despite the fact that in the last two decades several clinical trials on the topic of poor ovarian response have been made, so far it has been impossible to identify any efficient treatment to improve the ovarian response and the clinical outcome of this group of patients. Dehydroepiandrosterone (DHEA) is currently widely used worldwide and is considered a potential agent to improve the IVF outcomes of PORs. DHEA is an endogenous steroid generated by the adrenal glands and ovarian theca cells (7). Evidence shows that DHEA levels decrease with age (2). DHEA may influence ovarian follicular growth, not only by acting as a metabolic precursor for steroid production, but also by serving as ligands for androgen receptors (18). Indeed, androgen has been reported to play roles in recruitment and initiation of primordial follicles $(27,20)$, promotion of follicular growth by increasing FSH receptor expression, and prevention of follicular atresia by reducing apoptosis (26). DHEA could improve mitochondrial function and reduce apoptosis in the cumulus cells and human granulosa cell line (19). Another possible mechanism was described by Casson et al. (8), who described a transient increase in insulin-like growth factor 1 (IGF-1) in patients undergoing exogenous gonadotrophin ovulation induction after pre-treatment with DHEA. The IGF-I amplifies the effect of FSH 
at the level of both granulosa and theca cells $(10,29)$. This beneficial effect of IGF-1 has been reported to be correlated with oocyte quality and embryo development (13). Barad and Gleicher postulated that the effect of DHEA was due to the creation of polycystic ovarian syndrome (PCOS)-like characteristics in the aging ovary (4). Long-term androgen exposure can induce histological and sonographic changes in normal ovaries similar to PCOS (17). The effect of DHEA is cumulative as more of the antral follicles become exposed to treatment, as described by Barad and Gleicher (5). The theory of PCOS-like environment can explain the increase in response from cycle to cycle under DHEA exposure.

Patients receiving DHEA supplements should be informed of the potential negative side effects associated with DHEA, such as acne, oily skin, deepening of the voice, hirsutism and hair loss. Long-term effects of DHEA supplementation remain unknown. Possible risks include masculinized daughters and sex steroid dependent malignancies.

Metformin is a biguanide that lowers blood glucose levels in hyperglycemic individuals with type-2 diabetes mellitus but has no effect on glucose levels in normal subjects (3). Beginning in the 1990s, a series of studies indicated that metformin reduced insulin resistance in women with PCOS and increased the likelihood of ovulation and pregnancy (28). Hyperinsulinemia, as observed in PCOS subjects, may contribute to the aberrant response of granulosa cells to gonadotropins (9) and to the consequent arrest of follicle growth (12). However, the exact role of metformin in the management of women with PCOS has been quite controversial. Metformin modifies the ovarian morphology in PCOS (11), enhances intraovarian androgen levels (24), and improves systemic and local insulin resistance (25).

Therapy with metformin does not lead to weight gain and may be associated with modest weight loss, largely because of a slight anorectic effect as well as gastrointestinal side effects, including abdominal discomfort, diarrhea, nausea, and vomiting.

\section{Conclusion}

Our protocol seemed to improve the number of oocytes retrieved, clinical pregnancy rate and live birth rates in PORs. However, additional large-scale, well-designed randomized controlled trials are necessary to confirm the benefits of pretreatment with DHEA combining metformin in PORs.

\section{Disclosure of interests}

None declared. Completed disclosure of interests forms are available to view online as supporting information.

\section{Contribution to authorship}

Chang Chih-Chia substantial contributions to conception and design, final approval of the version to be published;Chang Yu-Jui acquisition of data, or analysis and interpretation of data;Chang Yu-Hsuan drafting the article or revising it critically for important intellectual content.

\section{Details of ethics approval}

Not required for this work

\section{Funding}

None

\section{Acknowledgements}

None

\section{References}

1. Amer, S. , 2007. Gonadotrophin induction of ovulation. Obstet. Gynaecol. Reprod. Med. 205-210.

2. Amirikia H, Savoy-Moore RT, Sundareson AS, Moghissi KS. The effects of long-term androgen treatment on the ovary. Fertil Steril 1986;45:202-8. 
3. Bailey CJ. Metformin revisited: its actions and indications for use. Diabet Med 1988;5:315-20.

4. Barad D, Gleicher N. Effect of dehydroepiandrosterone on oocyte and embryo yields, embryo grade and cell number in IVF. Hum Reprod 2006;21:2845-9.

5. Barad D, Gleicher N. Increased oocyte production after treatment with dehydroepiandrosterone. Fertil Steril 2005;84:756.

6. Biljan MM, Buckett WM, Dean N, Phillips SJ, Tan SL. The outcome of IVF-embryo transfer treatment in patients who develop three follicles or less. Hum Reprod 2000;15:2140-2144.

7. Burger HG. Androgen production in women. Fertil Steril. 2002;77(Suppl 4):S3-5.

8. Casson PR, Santoro N, Elkind-Hirsch K, Carson SA, Hornsby PJ, Abraham G, et al. Postmenopausal dehydroepiandrosterone administration increases free insulin-like growth factor-I and decreases high-density lipoprotein: a six-month trial. Fertil Steril 1998;70:107-10.

9. CofflerMS, Patel K, Dahan MH, Yoo RY,Malcom PJ, Chang RJ. Enhanced granulosa cell responsiveness to follicle-stimulating hormone during insulin infusion in women with polycystic ovary syndrome treated with pioglitazone. J Clin Endocrinol Metab 2003;88:5624-31.

10. E. Y. Adashi, C. E. Resnick, A. J. D'Ercole, M. E. Svoboda, and J. J. Van Wyk, "Insulin-like growth factors as intraovarian regulators of granulosa cell growth and function.," Endocrine Reviews, vol. 6, no. 3, pp. 400-420, 1985.

11. Falbo A, Orio F, Venturella R, Rania E, Materazzo C, Tolino A, et al. Does metformin affect ovarian morphology in patients with polycystic ovary syndrome? A retrospective cross-sectional preliminary analysis. J Ovarian Res 2009;31:2-5.

12. Franks S, Stark J, Hardy K. Follicle dynamics and anovulation in polycystic ovary syndrome. Hum Reprod Update 2008;14:367-78.

13. Fried G, Remaeus K, Harlin J, Krog E, Csemiczky G, Aanesen A, et al. Inhibin B predicts oocyte number and the ratio IGF-I/IGFBP-1 may indicate oocyte quality during ovarian hyperstimulation for in vitro fertilization. J Assist Reprod Genet. 2003;20 (5) :167-76.

14. Gleicher, N., Barad, D.H. Dehydroepiandrosterone (DHEA) supplementation in diminished ovarian reserve (DOR). Reprod. Biol. Endocrinol. 2011;9, 67.

15. Gleicher, N., Weghofer, A., Barad, D.H., 2011. The role of androgens in follicle maturation and ovulation induction: friend or foe of infertility treatment? Reprod. Biol. Endocrinol. 9, 116.

16. Gleicher, N., Kim, A., Weghofer, A., Kushnir, V.A., Shohat-Tal, A., Lazzaroni, E., Lee, H.J., Barad, D.H., 2013. Hypoandrogenism in association with diminished functional ovarian reserve. Hum. Reprod. 28, 1084-1091.

17. Harper AJ, Buster JE, Casson PR. Changes in adrenocortical function with aging and therapeutic implications. Semin Reprod Endocrinol 1999;17:327-38.

18. Hillier SG, Whitelaw PF, Smyth CD. Follicular estrogen synthesis: the '2-cell, two-gonadotrophin' model revisited. Mol Cell Endocrinol 1994;100:5-4.

19. Lin LT, Wang PH, Wen ZH, Li CJ, Chen SN, Tsai EM, et al. The application of Dehydroepiandrosterone on improving mitochondrial function and reducing apoptosis of cumulus cells in poor ovarian responders. Int J Med Sci. 2017;14(6):585-94.

20. Magamage MPS, Zengyo M, Moniruzzaman M, Miyano T. Testosterone induces activation of porcine primordial follicles in vitro. Reprod Med Biol. 2011;10(1):21-30. 
21. McGee, E.A., Hsueh, A.J., 2000. Initial and cyclic recruitment of ovarian follicles. Endocr. Rev. 21, 200-214.

22. Nestler JE, Jakubowicz DJ, Evans WS, Pasquali R. Effects of metformin on spontaneous and clomipheneinduced ovulation in the polycystic ovary syndrome. N Engl J Med 1998;338:1876-1880.

23. Orvieto R, Meltcer S, Nahum R, Rabinson J, Anteby EY, Ashkenazi J. The influence of body mass index on in vitro fertilization outcome. Int J Gynaecol Obstet 2009; 104:53-55.

24. Palomba S, Falbo A, Zullo F, Orio F. Evidence-based and potential benefits of metformin in the polycystic ovary syndrome: a comprehensive review. Endocr Rev 2009;30:1-50.

25. Palomba S, Falbo A, Russo T, Orio F, Tolino A, Zullo F. Systemic and local effects of metformin administration in patients with polycystic ovary syndrome (PCOS): relationship to the ovulatory response. Hum Reprod 2010;25:1005-13.

26. Sen A, Prizant H, Light A, Biswas A, Hayes E, Lee HJ, et al. Androgens regulate ovarian follicular development by increasing follicle stimulating hormone receptor and microRNA-125b expression. Proc Natl Acad Sci U S A. 2014;111(8):3008-13.

27. Smith P, Steckler TL, Veiga-Lopez A, Padmanabhan V. Developmental programming: differential effects of prenatal testosterone and dihydrotestosterone on follicular recruitment, depletion of follicular reserve, and ovarian morphology in sheep. Biol Reprod. 2009;80(4):726-36.

28. Velazquez EM, Mendoza S, Hamer T, Sosa F, Glueck DJ. Metformin therapy in polycystic ovary syndrome reduces hyperinsulinemia, insulin resistance, hyperandrogenemia, and systolic blood pressure, while facilitating normal menses and pregnancy. Metabolism 1994;43: 647-54.

29. X.-C. Jia, J. Kalmijn, and A. J. W. Hsueh, "Growth hormone enhances follicle

Stimulating hormone induced differentiation of cultured rat granulosa cells," Endocrinology, vol. 118, no. 4, pp. 1401-1409, 1986.

\section{Hosted file}

3. docx available at https://authorea.com/users/338531/articles/465030-dehydroepiandrosteronedhea-combining-metformin-adjuvant-agents-for-ovulation-induction-in-poor-ovarianresponders 